\title{
Avaliação do sistema API20C AUX na identificação de leveduras de interesse clínico
}

\author{
Evaluation of the API20C AUX system for the identification \\ of clinically important yeasts
}

\author{
Jaqueline Otero Silva ${ }^{1}$ Regina Célia Candido ${ }^{2}$
}

\begin{abstract}
RESUM0
Cinqüenta leveduras, pertencentes aos gêneros Candida, Pichia, Saccharomyces, Cryptococcus, Rhodotorula e Trichosporon, foram identificadas pelas metodologias clássica e sistema API20C AUX. O sistema comercial identificou corretamente $92 \%$ das espécies sendo necessários testes adicionais em 16\% dos casos. Os resultados foram interpretados como bom, muito bom e de excelente identificação.
\end{abstract}

Palavras-chaves: API 20C AUX. Candida sp. Leveduras. Trichosporon sp.

\begin{abstract}
Fifty yeasts belonging to the genera Candida, Pichia, Saccharomyces, Cryptococcus, Rhodotorula and Trichosporon were identified by classical methods and by the API 20C AUX system. The commercial system correctly identified $92 \%$ of the species, with the need for additional tests in $16 \%$ of cases. The results were interpreted with good, very good and excellent identification.
\end{abstract}

Key-words: API 20C AUX. Candida sp. Yeasts. Trichosporon sp.

As leveduras do gênero Candida podem causar uma variedade de síndromes em humanos, desde infecções superficiais até invasoras, principalmente em imunocomprometidos. Para compreender 0 prognóstico, a epidemiologia e a terapêutica é essencial que se identifique corretamente as diferentes espécies etiológicas.

A rotina para identificação das leveduras envolve além do exame da colônia e morfologia microscópica, várias reações bioquímicas. C. albicans, a espécie mais freqüente em materiais biológicos, tem sido identificada apenas pela produção de tubo germinativo em soro e produção de clamidosporos em ágar fubá acrescido de Tween 80. Outras espécies necessitam de provas de assimilação e fermentação de carbono que por sua vez consomem tempo, são trabalhosos e complexos ${ }^{35}$ apesar de alguns esquemas simplificados, baseados nesses princípios, terem sido propostos para identificação rápida de algumas delas 5 .

Atualmente são comercializados painéis manuais e automatizados para identificação de leveduras, através da capacidade assimilativa em substratos bioquímicos e enzimáticos os quais são de realização e interpretação fáceis, além de oferecer 0 resultado em tempo menor ${ }^{24678}$.

0 presente trabalho teve como objetivo avaliar o sistema comercial API20C AUX utilizando as diferentes espécies de leveduras previamente identificadas pela metodologia clássica.

Organismos testados. Selecionou-se para investigação, 50 leveduras incluindo os gêneros Candida, Pichia, Saccharomyces, Cryptococcus, Rhodotorula e Trichosporon obtidas de culturas de diversos materiais biológicos humanos. Todos os isolados foram testados pela metodologia clássica e reidentificadas pelo sistema API20C AUX.

Metodologia clássica: os isolados de C. albicans foram confirmados pela produção de tubo germinativo em soro bovino e produção de clamidosporos em ágar fubá acrescido de tween 80. Para exclusão de uma possível C. dubliniensis verificou-se a presença ou não de crescimento a $45^{\circ} \mathrm{C}$. Para identificação das outras espécies de Candida, utilizou-se além das características morfológicas em ágar fubá acrescido de tween 80, provas de assimilação e fermentação de diferentes carboidratos. Assimilação

\footnotetext{
1. Laboratório I de Ribeirão Preto do Instituto Adolfo Lutz, Ribeirão Preto, SP. 2. Departamento de Análises Clínicas, Bromatológicas e Toxicológicas da Faculdade de Ciências Farmacêuticas de Ribeirão Preto da Universidade de São Paulo, Ribeirão Preto, SP.

Endereço para correspondência: Dra. Jaqueline Otero Silva. R. Minas 877, Campos Elíseos, 14085-410 Ribeirão Preto, SP,

Tel: 5516 625-5046; Fax: 55 16, 635-7994

e-mail: jaquelineos@ig.com.br

Recebido para publicação em 10/3/2004

Aceito em 2/3/2005
} 
de nitrato, produção das enzimas urease e fenoloxidase foram utilizadas quando necessárias.

Sistema API 20C AUX: porção do crescimento de cada colônia bem isolada em ágar Sabouraud dextrose e de cultivo de 24-48 horas foi assepticamente transferida para tubos contendo $1 \mathrm{~mL}$ de solução fisiológica esterilizada e ajustado a uma turbidez equivalente à escala 2 de McFarland. Uma gota desta suspensão foi adicionada ao meio basal do API20C AUX e homogeneizado preenchendo, posteriormente, cada poço do painel de identificação. 0 painel foi incubado em câmara úmida, previamente preparada, a $30^{\circ} \mathrm{C}$, por até 72 horas, com leituras de 24,48 e 72 horas. Considerou-se como resultado positivo e negativo, respectivamente, a presença ou ausência de opacidade nos poços de cada carboidrato. Obteve-se um código de sete dígitos, cuja interpretação foi realizada com 0 auxílio do catálogo analítico API20C AUX. Identificações listadas no index como excelente ( $\%$ id $\geq 99,9$ e $\mathrm{T} \geq 0,75$ ), muito boa ( $\%$ id $\geq 99,0$ e $\mathrm{T} \geq 0,5$ ), ou aceitável (\%id $\geq 90,0$ e $\mathrm{T} \geq 0,5$ ), foram consideradas corretas ${ }^{1}$.

0 sistema API20C AUX identificou corretamente 92\% ( 46) das leveduras, sendo que 76\% (38) não necessitaram de testes adicionais e em $16 \%$ (8) foram necessários a inclusão dos mesmos. Resultados estes próximos aos de Sand e Rennie ${ }^{6}$ que encontraram 96,5\% de acurácia após 72 horas, quando da inclusão de testes extras. Melhores resultados foram obtidos por Smith eta ${ }^{8}$ que encontraram $95,6 \%$ de identificação sem adição de testes extras.

Foram observados problemas com a habilidade do sistema API20C AUX identificar corretamente C. krusei sem a necessidade de testes suplementares (Tabela 1). Wadlin et al ${ }^{9}$, também, encontraram dificuldades com 0 sistema, em identificar 23 isolados de C. krusei.

0 sistema comercial não identificou uma R. rubra ( $\mathrm{R}$. mucilaginosa) e uma C. zeylanoides, contudo uma Candida sp e um Trichosporon sp, não identificados como espécie pela metodologia clássica foram identificadas pelo sistema API20CAUX como C. rugosa e T. asahii, respectivamente (Tabela 1).

Smith et $\mathrm{al}^{8}$ encontraram dificuldade em identificar C. zeylanoides (3/3) pelo sistema API20C.

Foram obtidos os perfis numéricos de identificação, resultantes das reações bioquímicas desenvolvidas no sistema $\mathrm{e}$ analisados de acordo com 0 catálogo analítico ${ }^{1}$ (Tabela 2). As identificações da maioria das cepas foram interpretadas pelo sistema como boa, muito boa e excelente. Quando o sistema ofereceu a possibilidade de duas espécies (Tabela 2), as características morfológicas foram fundamentais para diferenciação das mesmas.

Smith eta ${ }^{8}$ encontraram superioridade do API20CAUX quando da comparação com o sistema RYP e concluíram que este fato foi devido à utilização do estudo morfológico em microcultivo, conforme recomendado pelo fabricante do sistema API 20C AUX.

Quanto aos dois isolados (C. coliculosa e C. utilis) que apresentaram resultados de identificação divergentes da metodologia clássica (respectivamente, C. bombii e C. fabianii) , observou-se que foram corretos quanto ao gênero.

Fenn et $\mathrm{al}^{2}$ verificaram acurácia de $100 \%$ do sistema API $20 \mathrm{C}$ em relação à identificação dos gêneros.

Na presente pesquisa, 0 sistema falhou em discriminar 0 gênero em duas ocasiões, respectivamente P. wicherhanii $x$

Talela 1 - Identificação das 50 leveduras pelo sistema API 20C AUX.

\begin{tabular}{|c|c|c|c|c|c|}
\hline Espécies & Avaliadas & $\begin{array}{l}\text { Corretamente } \\
\text { identificadas }\end{array}$ & $\begin{array}{c}\text { Identificadas com } \\
\text { testes extras }\end{array}$ & $\begin{array}{c}\text { Não } \\
\text { identificadas }\end{array}$ & $\begin{array}{c}\text { Incorretamente } \\
\text { identificadas } \\
\end{array}$ \\
\hline Candida albicans & 4 & 4 & - & - & - \\
\hline Candida parapsilosis & 6 & 6 & - & - & - \\
\hline Candida zeylanoides & 3 & 2 & - & 1 & - \\
\hline Candida krusei & 3 & - & 3 & - & - \\
\hline Candida tropicalis & 5 & 3 & 2 & - & - \\
\hline Candida glabrata & 6 & 5 & 1 & - & - \\
\hline Candida bombi & 1 & - & - & - & 1 \\
\hline Candida fabiani & 1 & - & - & - & 1 \\
\hline Candida guilliermondii & 4 & 4 & - & - & - \\
\hline \multicolumn{6}{|l|}{ Candida guilliermondii } \\
\hline var membranifaciens & 1 & - & 1 & - & - \\
\hline Candida spa & 1 & 1 & - & - & - \\
\hline Pichia anomala & 1 & - & 1 & - & - \\
\hline Saccaromyces cerevisae & 4 & 4 & - & - & - \\
\hline Cryptococcus albidus & 1 & 1 & - & - & - \\
\hline Rhodotorula rubrab & 4 & 3 & - & 1 & - \\
\hline Rhodotorula. minuta & 1 & 1 & - & - & - \\
\hline Trichosporon asahii & 1 & 1 & - & - & - \\
\hline Trichosporon inkin & 1 & 1 & - & - & - \\
\hline Trichosporon mucoides & 1 & 1 & - & - & - \\
\hline Trichosporon $\mathrm{sp}^{\mathrm{c}}$ & 1 & 1 & - & - & - \\
\hline Total & 50 & 38 & 8 & 2 & 2 \\
\hline
\end{tabular}

consideradas como ${ }^{a}$ Candida rugosa; ${ }^{b}$ Rhodotorula mucilaginosa; ${ }^{\circ}$ Trichosporon asahii em data base do sistema. 
Tabela 2 - Perfil de identificação das leveduras através do sistema API20C AUX.

\begin{tabular}{|c|c|c|c|c|}
\hline \multirow{2}{*}{$\begin{array}{l}\text { Identificação clássica } \\
\text { Espécie }\left(\mathrm{n}^{0}\right)\end{array}$} & \multicolumn{3}{|c|}{ Sistema API20C AUX } & \multirow[b]{2}{*}{ acerto $(\%)$} \\
\hline & perfil numérico & espécie & cepas $n^{0}$ & \\
\hline \multirow[t]{3}{*}{ Candida albicans (4) } & 2576174 & Candida albicans (1) & 2 & 97,4 \\
\hline & 6576174 & Candida albicans (1) & 1 & 98,2 \\
\hline & 2542134 & Candida albicans (2) & 1 & 97,4 \\
\hline \multirow[t]{3}{*}{ Candida parapsilosis (6) } & 6756175 & Candida parapsilosis & 4 & 99,9 \\
\hline & 6552135 & Candida parapsilosis & 1 & 99,9 \\
\hline & 6752135 & Candida parapsilosis & 1 & 99,7 \\
\hline \multirow[t]{2}{*}{ Candida zeylanoides (3) } & 6102140 & Candida zeylanoides & 2 & 99,9 \\
\hline & 6752344 & não identificada & 1 & \\
\hline Candida krusei (3) & 6000104 & Candida krusei / inconspícua & 3 & 96,3 \\
\hline \multicolumn{5}{|l|}{ Candida guilliermondii } \\
\hline \multirow[t]{4}{*}{ var. guilliermondii (4) } & 6776376 & Candida. guilliermondii & 1 & 99,6 \\
\hline & 6776277 & Candida guilliermondii & 1 & 99,6 \\
\hline & 6736377 & Candida guilliermondii & 1 & 99,7 \\
\hline & 6756377 & Candida guilliermondii & 1 & 98,9 \\
\hline \multicolumn{5}{|l|}{ Candida guilliermondii } \\
\hline var. membranifaciens (1) & 6146376 & Pichia ohmeri & 1 & 99,9 \\
\hline Candida bombi (1) & 610062 & Candida coliculosa & 1 & 96,9 \\
\hline Candida fabiani (1) & 6400237 & Candida utilis & 1 & 99,8 \\
\hline Candida sp (1) & 6462004 & Candida rugosa/Candida boidinii & 1 & $77,9 / 21,2$ \\
\hline \multirow[t]{2}{*}{ Candida tropicalis (5) } & 2556175 & Candida tropicalis & 3 & 95,9 \\
\hline & 2556375 & Candida tropicalis ou Candida lusitaniae & 2 & $89,4-10,5$ \\
\hline \multirow[t]{2}{*}{ Candida glabrata (6) } & 2000040 & Candida glabrata & 5 & 99,4 \\
\hline & 6000040 & Candida glabrata ou Pichia wicherhanii & 1 & $29,3-70,7$ \\
\hline Cryptococcus albidus (1) & 2704273 & Cryptococcus albidus & 1 & 99,9 \\
\hline Pichia anomala (1) & 6404071 & Candida peliculosa & 1 & 98,2 \\
\hline \multirow[t]{4}{*}{ Saccaromyces cerevisae (4) } & 2040014 & Saccaromyces cerevisae & 1 & 99,4 \\
\hline & 2044073 & Saccaromyces cerevisae & 1 & 99,9 \\
\hline & 2040022 & Saccaromyces cerevisae & 1 & 81,3 \\
\hline & 2040032 & Saccaromyces cerevisae & 1 & 98,7 \\
\hline \multirow[t]{3}{*}{ Rhodotorula rubra (4) } & 6640073 & Rhodotorula mucilaginosa (2) & 2 & 99,9 \\
\hline & 2642073 & Rhodotorula mucilaginosa (2) & 1 & 99,9 \\
\hline & 6640033 & não identificada & 1 & - \\
\hline Rhodotorula minuta (1) & 6700060 & Rhodotorula minuta & 1 & 99,9 \\
\hline Trichosporon asahii (1) & 2744774 & Trichosporon asahii & 1 & 99,9 \\
\hline Trichosporon inkin (1) & 2545775 & Trichosporon inkin & 1 & 99,9 \\
\hline Trichosporon mucoides (1) & 6745776 & Trichosporon mucoides ou Cryptococcus humicolus & 1 & $67,3-32,1$ \\
\hline Trichosporon sp (1) & 2744775 & Trichosporon asahii & 1 & 99,9 \\
\hline
\end{tabular}

C. glabrata e T. mucoides x C. humicolus, sendo a morfologia macroscópica primordial na identificação (Tabela 2) .

0 sistema comercial API20C AUX ofereceu todas as vantagens de armazenamento, estabilidade, reprodutibilidade e facilidade de uso oferecendo resultados em 72 horas. Porém, apresenta limitações por depender muitas vezes de provas suplementares para completa identificação.

\section{REFERÊNCIAS BIBLIOGRÁFICAS}

1. Catalogue Analytique: API 20C AUX, 3a edição Biomerrieux, 1997.

2. Fenn JP, Segal H, Barland B, Denton D, Whisenant J, Chun H, Christofferson K, Hamilton L, Carrol K. Comparison of update Vitek yeast biochemical card and API 20C yeastidentification systems. Journal Clinical Microbiology 32: 1184-1187, 1994.

3. Lacaz CS, Porto E, Martins JEC. Micologia Médica 8a edição São Paulo Editora Sarvier, São Paulo, p.175-176, 187-190, 546, 561, 557, 586, 1991.
4. Land GA, Harrison BA, Hulme KL, Cooper BH, Byrd JC. Evaluation of the new API 20C strip for yeast identification agaist a conventional method. Journal Clinical Microbiology 10: 357-364, 1979.

5. Lopes J, Dalle F, Mantelin P, Moiroux P, Nierlich AC, Pacot A, Cuisenier B, Vagner 0, Bonnin A. Rapid identification of Candida glabrata based on trealose and sucrose assimilation using Rosco Diagnostic Tablets. Journal Clinical Microbiology 39:1172-1174, 2001.

6. Sand C, Rennie RP. Comparison of three commercial systems for the identification of germ-tube negative yeast species isolated from clinical specimens. Diagnostic Microbiology Infectious Diseases 33: 223-229, 1999.

7. Sheppard DC, René $P$, Harris $A D$, Miller MA, Laverdière M, Souza E, Robson HG. Simple strategy for direct identification of medically important yeast species from positive blood culture vials. Journal Clinical Microbiology 37: 2040-2041, 1999.

8. Smith MB, Dunklee D, Hangna Vu, Woods GL. Comparative performance of the RapID yeast plus system and the API 20C AUX clinical yeast system. Journal Clinical Microbiology 37: 2697-2698, 1999.

9. Wadlin JK, Hanko G, Stewart R, Pape J, Nachamkin I. Comparison of three commercial systems for identification of yeasts commonly isolated in the clinical microbiology laboratory. Journal Clinical Microbiology 37: 1967-1970, 1999. 